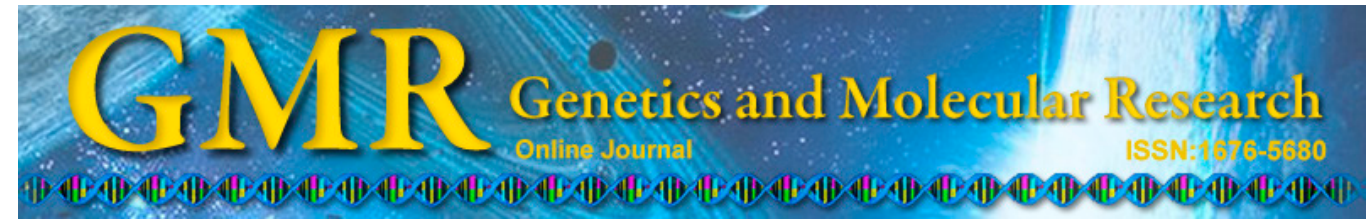

\title{
Genome-wide transcriptional profiling reveals molecular signatures of secondary xylem differentiation in Populus tomentosa
}

\author{
X.H. Yang ${ }^{1,2}$, X.G. Li ${ }^{3}$, B.L. Li ${ }^{1,2,4}$ and D.Q. Zhang ${ }^{1,2}$ \\ ${ }^{1}$ National Engineering Laboratory for Tree Breeding, \\ College of Biological Sciences and Technology, Beijing Forestry University, \\ Beijing, China \\ ${ }^{2}$ Key Laboratory of Genetics and Breeding in Forest Trees and Ornamental Plants, \\ Ministry of Education, College of Biological Sciences and Technology, \\ Beijing Forestry University, Beijing, China \\ ${ }^{3}$ CSIRO Plant Industry, Canberra ACT, Australia \\ ${ }^{4}$ Department of Forestry, North Carolina State University, Raleigh, NC, USA \\ Corresponding author: D.Q. Zhang \\ E-mail: DeqiangZhang@bjfu.edu.cn
}

Genet. Mol. Res. 13 (4): 9489-9504 (2014)

Received October 17, 2013

Accepted May 27, 2014

Published November 11, 2014

DOI http://dx.doi.org/10.4238/2014.November.11.14

ABSTRACT. Wood formation occurs via cell division, primary cell
wall and secondary wall formation, and programmed cell death in
the vascular cambium. Transcriptional profiling of secondary xylem
differentiation is essential for understanding the molecular mechanisms
underlying wood formation. Differential gene expression in secondary
xylem differentiation of Populus has been previously investigated
using cDNA microarray analysis. However, little is known about the
molecular mechanisms from a genome-wide perspective. In this study,
the Affymetrix poplar genome chips containing 61,413 probes were used
to investigate the changes in the transcriptome during secondary xylem
differentiation in Chinese white poplar (Populus tomentosa). Two xylem
tissues (newly formed and lignified) were sampled for genome-wide 
transcriptional profiling. In total, 6843 genes $(\sim 11 \%)$ were identified with differential expression in the two xylem tissues. Many genes involved in cell division, primary wall modification, and cellulose synthesis were preferentially expressed in the newly formed xylem. In contrast, many genes, including 4-coumarate:cinnamate-4-hydroxylase $(\mathrm{C} 4 \mathrm{H})$, 4-coumarate:CoA ligase (4CL), cinnamyl alcohol dehydrogenase (CAD), and caffeoyl CoA 3-O-methyltransferase (CCoAOMT), associated with lignin biosynthesis were more transcribed in the lignified xylem. The two xylem tissues also showed differential expression of genes related to various hormones; thus, the secondary xylem differentiation could be regulated by hormone signaling. Furthermore, many transcription factor genes were preferentially expressed in the lignified xylem, suggesting that wood lignification involves extensive transcription regulation. The genome-wide transcriptional profiling of secondary xylem differentiation could provide additional insights into the molecular basis of wood formation in poplar species.

Key words: Wood formation; Affymetrix microarrays; Secondary xylem differentiation; Lignified xylem; Vascular cambium; Newly formed xylem

\section{INTRODUCTION}

Tree growth consists of elongation growth originating from the shoot apical buds (Cutter, 1965) and periclinal growth driven by the vascular cambium (Larson, 1994). The vascular cambium is a group of meristematic cells derived from procambial cells formed during primary growth. The division of cambial initials produces xylem mother cells (Larson, 1994), which then develop primary and secondary xylem (wood). Formation of wood includes a series of developmental processes, such as cell division, primary cell wall synthesis, secondary wall formation, and programmed cell death (Hertzberg et al., 2001).

The molecular basis of wood formation has been previously studied in many forest tree species by using cDNA microarray analysis (Sterky et al., 1998; Li et al., 2009b), Affymetrix DNA chips (Bao et al., 2009), and RNA sequencing (Ramsköld et al., 2012). These studies have identified many genes that are differentially expressed in various wood developmental stages and highlighted the complex molecular mechanisms involved in wood formation. Furthermore, the sequenced genomes of Populus trichocarpa (Tuskan et al., 2006) and Picea abies (Norway spruce; Nystedt et al., 2013) provide fundamental basis for furthering study on wood formation in forest tree species.

The Chinese white poplar (Populus tomentosa Carr.) is the most important poplar species widely planted in commercial forests in Northern China. It plays a major role in the furniture and paper industries and territorial environmental protection. Previous studies on this species have mainly focused on the genes or gene families related to various stress resistances. Only one study investigated wood formation in this species at the transcriptional level by using cDNA microarray analysis of about 3000 clones (Wang et al., 2009). While they identified many genes that are highly expressed in the secondary xylem, knowledge on the genome-wide transcriptome changes during secondary xylem differentiation in Chinese white poplar and 
other poplar species remains largely limited. In this study, the Affymetrix poplar genome microarrays containing 61,413 probes were used to compare the xylem trancriptomes of Chinese white poplar during xylem differentiation. This study aimed to identify genes that are differentially expressed in the newly formed and lignified xylem from a genome-wide perspective.

\section{MATERIAL AND METHODS}

\section{Plant materials and sampling}

Five-two-year-old individuals of a $P$. tomentosa clone were selected for sampling. These trees were grown under normal conditions at Xiaotangshan, Beijing, China. Developing xylem tissues were sampled in early July (summer). A piece of bark (approximately $5 \mathrm{~cm}$ in length) was first removed from each tree at about $1 \mathrm{~m}$ high above the ground. The newly formed xylem (approximately $0.5 \mathrm{~mm}$ deep) was collected from the exposed surface by using a knife. The xylem was further scraped at a depth of 1-2 $\mathrm{mm}$ toward the inner xylem, representing lignified xylem. These samples were immediately placed in separate Falcon ${ }^{\mathrm{TM}}$ tubes filled with liquid nitrogen, and then stored in a freeze at $-80^{\circ} \mathrm{C}$ until RNA extraction. All samples were uploaded at the Gene Expression Omnibus (http://www.ncbi.nlm.nih.ov/geo/) with the accession number GSE46946.

\section{RNA extraction}

The two developing xylem samples collected from the five trees were pooled separately and then divided into three subsamples for RNA extraction. RNAs were extracted using the RNeasy plant mini kits (Qiagen, Inc., Valencia, CA, USA). The quality of RNAs was checked by performing agarose gel electrophoresis and reading absorbance. The $18 \mathrm{~s} / 28 \mathrm{~s}$ ratios

of all RNA samples ranged from 1.3 to 1.8 , and the $\mathrm{A}_{260 / 280}$ ratios were 1.8-2.1, indicating good quality for downstream use.

\section{Microarray platform and array hybridization}

The Affymetrix Poplar Genome GeneChip consisted of 61,413 probes representing over 56,000 transcripts. Microarray hybridization was performed at the Shanghai Bioarray Co. Ltd., according to Affymetrix ${ }^{\mathcal{O}}$ protocols. Signals of the hybridized arrays were screened using an Affymetrix scanner. Raw gene expression data were read and analyzed using the Affymetrix GeneChip ${ }^{\circledR}$ Operating Software. The quality of data was verified using a list of parameters such as probe array image inspection, B2 oligo performance, average background, noise values, poly-A controls, hybridization controls, internal control genes, percent present, scaling, and normalization factors (Bao et al., 2009).

\section{Gene filtering and function annotation}

Differentially expressed genes were selected based on a 2-fold difference of gene expression between the newly formed and lignified xylem $\left(\log _{2}\right.$ ratios $\geq 1$ or $\left.\leq-1\right)$ with $P$ values of $\leq 0.01$ for statistical significance. The identified genes were annotated using the Affymetrix Poplar Chip annotation (against JGI release v2.2; Tuskan et al., 2006); Uniprot 
(http://www.uniprot.org/uniprot); gene models of poplar (Tuskan et al., 2006); Arabidopsis (TAIR10; http://www.arabidopsis.org/); rice (Ouyang et al., 2007); and medicago (Li et al., 2009a), as well as Gene Ontology (GO) terms (http://www.geneontology.org/). The probes without a poplar gene model were also annotated using the GenBank accession numbers and the poplar predicted gene set v2.2 (http://www.phytozome.net/poplar; Tuskan et al., 2006). AgriGO (Du et al., 2010) was used to identify genes related to biological processes and cellular components. Genes involved in cell wall formation were identified using the Cell Wall Navigator (http://bioweb.ucr.edu/Cellwall/; Girke et al., 2004). Transcription factors were identified using the Plant TFDB (Zhang et al., 2011). Heat map of differential gene expression was generated using R (R Core Team, 2012).

\section{Real-time reverse transcription-polymerase chain reaction (RT-PCR)}

Eight genes were chosen from the differentially expressed genes identified in the microarray analysis and used for the validation by using real-time RT-PCR. These genes included phosphatase 2C (POPTR_0013s01370), patatin-like protein 9 (POPTR_0005s23170), basic chitinase (CV277108), an unknown protein (POPTR_0008s14970), thaumatin (POPTR_0009s13510), expansin-A4 (POPTR_0008s05720), expansin-A1 (POPTR_0008s08790), and expansin-A8 (POPTR_0013s15080). Primers were designed with a melting temperature of $58^{\circ} \mathrm{C}$ and products around 58-77 bp in length (Table S1). The amplification reaction was set in $25 \mu \mathrm{L}$ consisting of $1 \mathrm{X}$ SYBR Premix Ex Taq ${ }^{\mathrm{TM}}, 5 \mu \mathrm{M}$ of each primer, and 5 ng cDNA. RT-PCR was run using an Opticon2 thermocycler real-time PCR machine (BioRad, USA). The cycling parameters were set as follows: $2 \mathrm{~min}$ at $50^{\circ} \mathrm{C}, 5$ min at $94^{\circ} \mathrm{C}, 50$ cycles of $30 \mathrm{~s}$ at $94^{\circ} \mathrm{C}, 30 \mathrm{~s}$ at $58^{\circ} \mathrm{C}$, and $30 \mathrm{~s}$ at $72^{\circ} \mathrm{C}$, and a final elongation for $10 \mathrm{~min}$ at $72^{\circ} \mathrm{C}$. The specificity of the RT-PCR products was checked by performing a melting curve program from 70 to $95^{\circ} \mathrm{C}$ with holding for $10 \mathrm{~s}$ at each $0.5^{\circ} \mathrm{C}$. Fluorescence signals were detected at $58^{\circ} \mathrm{C}$ during the melting curve program. ACTIN (GenBank accession: AY261523.1) was used as a reference gene. Each sample was run three times, and data were analyzed using the Opticon Monitor Analysis 3.1 software (BioRad).

\section{RESULTS AND DISCUSSION}

\section{Xylem transcriptome changed from newly formed to lignified xylem}

While comparing between the newly formed and lignified xylem, of the 61,413 probes in the microarrays, 3134 were found to be preferentially expressed in the newly formed xylem, and 3709 were more highly expressed in the lignified xylem. This indicated that only a small proportion of the poplar xylem transcriptome (approximately 11\%) had differential expression during secondary xylem differentiation. Almost all the identified genes with differential expression $(95.3 \%$ for newly formed xylem and $93.7 \%$ for lignified xylem) had homologs in the sequenced poplar genome (Tuskan et al., 2006). Of the 3134 probes preferentially expressed in the newly formed xylem, $77.5 \%$ had homologs in Uniprot, and $94.8 \%$ had homologs in the TAIR databases. On the other hand, 69.1 and $92.3 \%$ of the 3709 probes for the lignified xylem had significant matches in the two public databases, respectively. 


\section{Functional annotation and classification of differentially expressed genes}

Differentially expressed genes were functionally annotated using the GO terms (Table 1). The majority of these genes $(80.4 \%$ for newly formed xylem and $79.7 \%$ for lignified xylem) had a role in biological process. Genes involved in translation (GO: 0006412), cell wall organization (GO: 0071555), cellulose biosynthetic process (GO: 0030244), and cytoskeleton organization (GO: 0007010) were highly transcribed in the newly formed xylem. On the other hand, genes with a role in transcription (GO: 0006355), hormone stimulus (GO: 0009725), and protein metabolism (GO: 0019538) were preferentially expressed in the lignified xylem. In molecular function, genes functioning in the structural constituents of ribosome (GO: 0003735), hydrolase activity (GO: 0004553), cellulose synthase (UDP-forming; GO: 0016760), and actin binding (GO: 0003779) were preferentially expressed in the newly formed xylem; on the other hand, genes involved in transcription activity (GO: 0003700), zinc-ion binding (GO: 0008270), and nucleic acid binding (GO: 0003676) were relatively highly expressed in the lignified xylem. In cellular component, genes participating in ribosome (GO: 0005840), cytoskeleton (GO: 0005856), and cell junction (GO: 0030054) were more abundant in the newly formed xylem whereas the genes as components in the nucleus and peroxisomal membrane were preferentially expressed in the lignified xylem.

\begin{tabular}{|c|c|c|c|}
\hline \multirow[t]{2}{*}{ Category } & \multirow[t]{2}{*}{ GO term } & \multicolumn{2}{|c|}{ Number of genes } \\
\hline & & Newly formed xylem & Lignified xylem \\
\hline$\overline{\mathrm{BP}}$ & GO:0006412|translation & 94 & 15 \\
\hline $\mathrm{BP}$ & GO:0006886|intracellular protein transport & 33 & 4 \\
\hline $\mathrm{BP}$ & GO:0051258|protein polymerization & 29 & 1 \\
\hline BP & GO:0007018|microtubule-based movement & 25 & 3 \\
\hline BP & GO:0071555|cell wall organization & 10 & 1 \\
\hline $\mathrm{BP}$ & GO:0042545 cell wall modification & 7 & 1 \\
\hline $\mathrm{BP}$ & GO:0030244|cellulose biosynthetic process & 15 & \\
\hline BP & GO:0006414|translational elongation & 11 & \\
\hline BP & GO:0007010|cytoskeleton organization & 7 & \\
\hline $\mathrm{BP}$ & GO:0006355|"regulation of transcription, DNA-dependent" & 36 & 124 \\
\hline BP & GO:0009725|response to hormone stimulus & 3 & 16 \\
\hline BP & GO:0019538|protein metabolic process & 30 & 68 \\
\hline MF & GO:0003735|structural constituent of ribosome & 99 & 13 \\
\hline MF & GO:0005525|GTP binding & 80 & 18 \\
\hline MF & GO:0004553|hydrolase activity & 69 & 22 \\
\hline MF & GO:0003924|GTPase activity & 50 & 5 \\
\hline MF & GO:0016760|cellulose synthase (UDP-forming) activity & 12 & \\
\hline MF & GO:0004634|phosphopyruvate hydratase activity & 9 & \\
\hline MF & GO:0003779|actin binding & 8 & \\
\hline MF & GO:0003700|transcription factor activity & 112 & 312 \\
\hline MF & GO:0008270|zinc ion binding & 53 & 145 \\
\hline MF & GO:0003676 nucleic acid binding & 32 & 86 \\
\hline MF & GO:0009055 electron carrier activity & 28 & 64 \\
\hline MF & GO:0003723|RNA binding & 19 & 44 \\
\hline $\mathrm{CC}$ & GO:0005840|ribosome & 99 & 13 \\
\hline $\mathrm{CC}$ & GO:0005737|cytoplasm & 40 & 9 \\
\hline $\mathrm{CC}$ & GO:0043234|protein complex & 29 & 1 \\
\hline $\mathrm{CC}$ & GO:0005783|endoplasmic reticulum & 21 & 3 \\
\hline $\mathrm{CC}$ & GO:0030131 clathrin adaptor complex & 14 & \\
\hline $\mathrm{CC}$ & GO:0000139|Golgi membrane & 10 & \\
\hline $\mathrm{CC}$ & GO:0000015 phosphopyruvate hydratase complex & 9 & \\
\hline $\mathrm{CC}$ & GO:0005856 cytoskeleton & 8 & \\
\hline $\mathrm{CC}$ & GO:0030054|cell junction & 3 & \\
\hline $\mathrm{CC}$ & GO:0005634|nucleus & 39 & 114 \\
\hline $\mathrm{CC}$ & GO:0005778 peroxisomal membrane & & 5 \\
\hline
\end{tabular}

$\mathrm{BP}=$ biological process; $\mathrm{MF}=$ molecular function $; \mathrm{CC}=$ cellular component . 
In the GO terms with biological process, genes involved in lignin metabolic process were more enriched in the lignified xylem (Figure 1). In contrast, genes involved in cytoskeleton were considerably more enriched in the newly formed xylem (Figure 2). The identified differentially expressed genes were further classified into 16 functional groups (Figure 3). Genes with hydrolase activity, kinase activity, microtubule motor activity, structural constituents of ribosomes, structural molecule activity, and transferase activity were more highly expressed in the newly formed xylem. On the other hand, genes with DNA binding, nucleic acid binding, RNA binding, transcription factor activity, and unknown functions were up-regulated in the lignified xylem. These results indicated that the two stages of xylem differentiation had significant differences in their xylem transcriptomes.

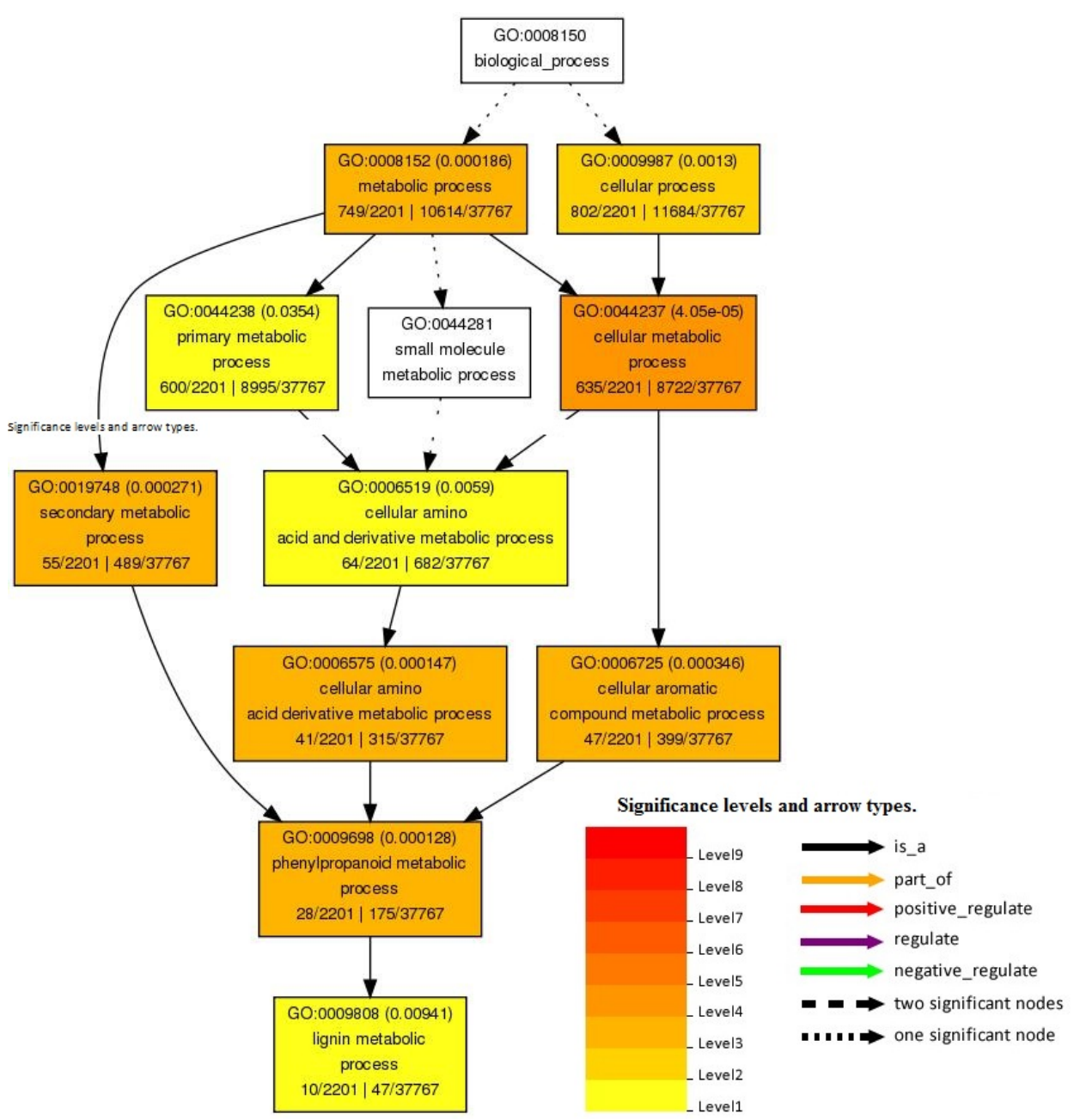

Figure 1. Gene Ontology (GO) terms of lignin metabolic process were significantly enriched in the lignified xylem. the AgriGO software was used for the analysis with $\mathrm{P}$ values $<0.05$ for statistical significances. The significant GO term node is presented by proportional color, as indicated by the scale. 


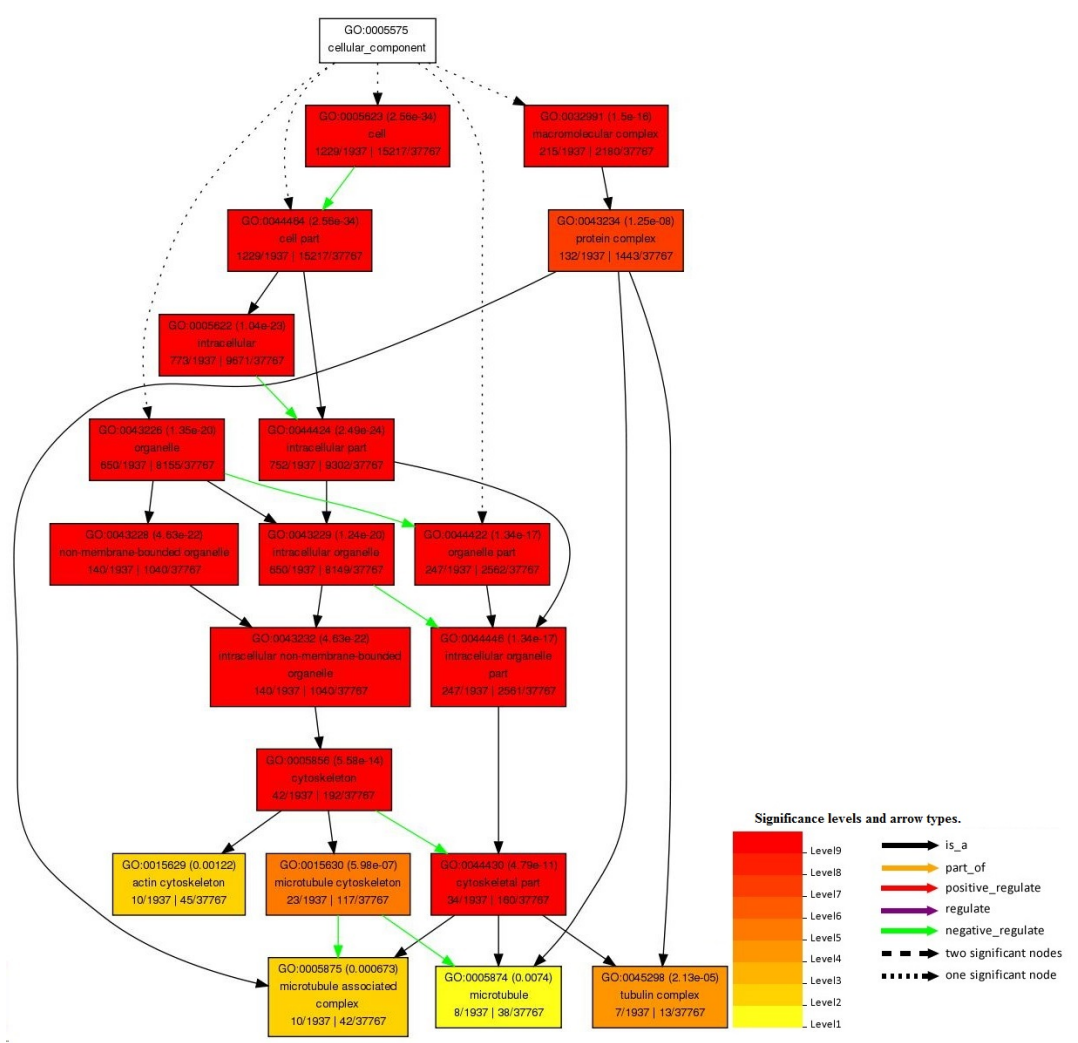

Figure 2. Gene Ontology (GO) terms of cytoskeleton in "Cellular Component" were significantly enriched in the newly formed xylem. The AgriGO software was used for the analysis with $\mathrm{P}$ values $<0.05$ for statistical significances. The significant GO term node is presented by proportional color, as indicated by the scale.

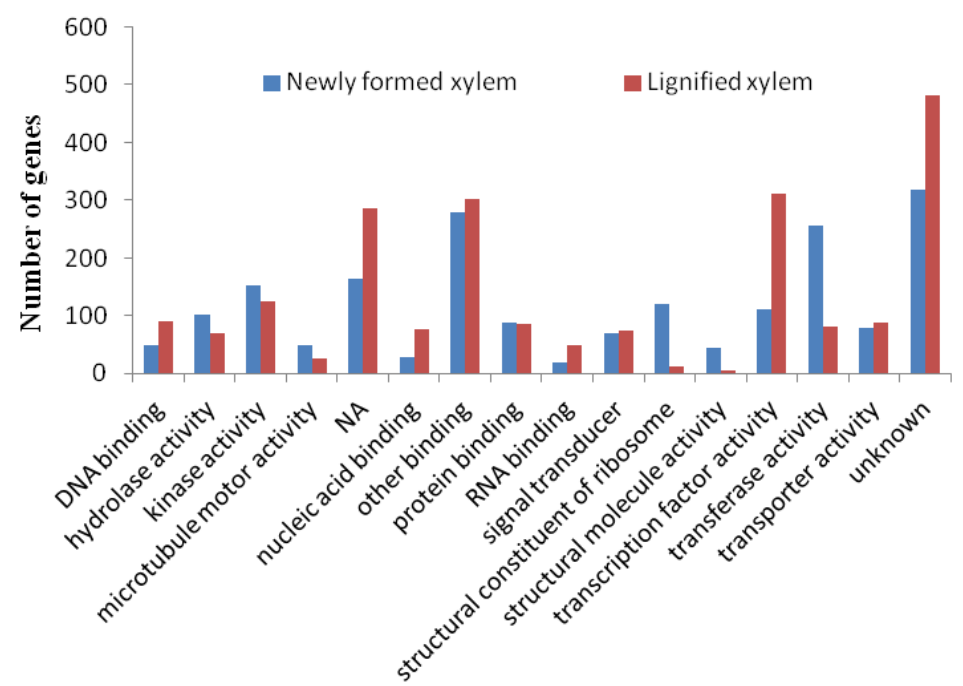

Figure 3. Functional categories of genes differentially expressed in the newly formed and lignified xylem. 


\section{Differential expression of cell wall-related genes}

Many differentially expressed genes were related to cytoskeleton, cell wall structural proteins (Table 2), and cell wall biosynthesis (Table 3 and Figure 4). Red and green colors indicate higher and lower transcript levels, respectively. The JGI gene models are shown on the right. $A$ and $B$ represent replicates 1 and 2 of the newly formed xylem, respectively; $G$ and $H$ indicate replicates 1 and 2 of the lignified xylem, respectively. Genes involved in cytoskeleton development were exclusively up-regulated in the newly formed xylem, such as genes encoding actins, tubulins, and tubulin-folding cofactor. Actins and tubulins are important units of actin microfilaments and microtubules, respectively (Oakley et al., 2007). Li et al. (2009b) found that the actin genes were highly abundant in the radiate pine xylem expressed sequence tags, indicating their possible function in wood formation. Microtubules play an important role in cellulose microfibril arrangement and deposition, and tubulins were highly expressed in the xylem (Li et al., 2009b).

\begin{tabular}{|c|c|c|c|}
\hline Gene & Poplar gene model & TAIR annotation & $\begin{array}{c}\log _{2} \text { ratios } \\
\text { (newly formed xylem/lignified xylem) }\end{array}$ \\
\hline$\overline{\text { Actin } 7}$ & POPTR_0001s31700 & AT5G09810 & -1.38 \\
\hline Actin-11 & POPTR_0006s 20710 & AT3G12110 & -3.52 \\
\hline Tubulin beta- 5 & POPTR_0002s 02340 & AT1G20010 & -4.09 \\
\hline Tubulin alpha-2 & POPTR_0009s 08850 & AT1G50010 & -1.28 \\
\hline Tubulin beta-7 & POPTR_1455s 00210 & AT2G29550 & -3.44 \\
\hline Tubulin beta 6 & POPTR_0001s09330 & AT5G12250 & -2.44 \\
\hline Tubulin beta 8 & POPTR_0006s09610 & AT5G23860 & -2.74 \\
\hline Tubulin beta 3 & POPTR_0001s27960 & AT5G62700 & -1.54 \\
\hline$A G P 18$ & POPTR_0005s 18840 & AT4G37450 & -3.21 \\
\hline$A G P 20$ & POPTR_0014s09050 & АT3G61640 & -3.10 \\
\hline$A G P 30$ & POPTR $0011 \mathrm{~s} 05340$ & AT2G33790 & -2.68 \\
\hline FLA 11 & POPTR_0012s02220 & AT5G03170 & 1.51 \\
\hline FLA 12 & POPTR_0013s14790 & AT5G60490 & 2.17 \\
\hline FLA 1 & POPTR_0001s37650 & AT5G55730 & -2.35 \\
\hline FLA 4 & POPTR_0006s 18920 & AT3G46550 & -1.08 \\
\hline FLA 6 & POPTR_0013s 12490 & AT2G20520 & -1.51 \\
\hline FLA 7 & POPTR_0014s 16100 & AT2G04780 & -3.89 \\
\hline FLA 10 & POPTR_0014s06740 & AT3G60900 & -3.12 \\
\hline FLA 17 & POPTR_0008s01310 & AT5G06390 & -1.65 \\
\hline Proline-rich protein & POPTR_0008s06680 & AT2G40070 & -1.21 \\
\hline Glycine-rich protein & POPTR_0008s 19880 & AT1G27090 & -1.02 \\
\hline Glycine-rich protein & POPTR_0011s04480 & AT4G21620 & -2.38 \\
\hline Glycine-rich protein & POPTR_0004s13090 & AT5G39570 & -2.05 \\
\hline Glycine-rich protein & POPTR_0011s03530 & AT1G11440 & 4.05 \\
\hline Glycine-rich protein & POPTR_0004s03670 & AT1G61255 & 3.52 \\
\hline Glycine-rich protein & POPTR_0006s 12580 & AT2G43630 & 1.24 \\
\hline$H R G P$ & POPTR_0012s12920 & AT5G52430 & 2.24 \\
\hline$H R G P$ & POPTR_0002s 10890 & AT5G65660 & 2.94 \\
\hline$H R G P$ & POPTR_0006s 25030 & AT2G25930 & 1.48 \\
\hline$H R G P$ & POPTR_0017s09000 & AT2G33490 & 1.04 \\
\hline$H R G P$ & POPTR_0008s 13830 & AT1G14710 & 3.08 \\
\hline$H R G P$ & POPTR_0005s 28120 & AT1G76660 & -2.13 \\
\hline$H R G P$ & POPTR_0017s 12970 & АТЗG02120 & -3.94 \\
\hline$H R G P$ & POPTR_0001s46460 & АТЗ 25690 & -2.49 \\
\hline$H R G P$ & POPTR_0004s 22000 & AT3G45230 & -2.40 \\
\hline$H R G P$ & POPTR_0009s01910 & AT3G45230 & -3.25 \\
\hline$H R G P$ & POPTR_0006s 24500 & AT5G11890 & -1.53 \\
\hline
\end{tabular}

$\mathrm{AGP}=$ arabinogalactan protein; FLA $=$ FASCICLIN-like arabinogalactan; HRGP $=$ hydroxyproline-rich glycoprotein family. $\log _{2}$ ratios represent relative expression of a gene between the lignified xylem and the newly formed xylem. Positive values indicate genes preferentially expressed in the lignified xylem, whereas negative values indicate genes preferentially expressed in the newly formed xylem. 
Table 3. Some cell wall-related genes preferentially expressed in the newly formed and lignified xylem.

\begin{tabular}{|c|c|c|c|}
\hline Gene & Poplar gene model & TAIR annotation & $\begin{array}{c}\log _{2} \text { ratios } \\
\text { (newly formed xylem/lignified xylem) }\end{array}$ \\
\hline Expansin Al & POPTR_0010s17440 & AT1G69530 & -3.84 \\
\hline Expansin $A 4$ & POPTR_0008s05720 & AT2G39700 & -3.16 \\
\hline Expansin-like A2 & POPTR_0004s 18840 & AT4G38400 & -3.64 \\
\hline Pectin lyase-like & POPTR_0014s10770 & AT3G62110 & -3.64 \\
\hline Pectin lyase-like & POPTR_-0003s 17450 & AT4G13710 & -3.62 \\
\hline Pectin lyase-like & POPTR $0015 \mathrm{~s} 09930$ & AT5G63180 & -4.33 \\
\hline$P M E 3$ & POPTR_0003s07040 & AT3G14310 & -3.31 \\
\hline PME 44 & POPTR_0006s 13670 & AT4G33220 & -5.24 \\
\hline$P A E$ & POPTR_0005s00330 & AT3G05910 & -3.92 \\
\hline$P A E$ & POPTR_0003s03720 & AT4G19410 & -4.26 \\
\hline$P A E$ & POPTR_0012s13090 & AT5G23870 & -3.31 \\
\hline Plant invertase/PME & POPTR_0006s 13680 & AT2G26440 & -3.87 \\
\hline Plant invertase/PME & POPTR_0012s14560 & AT5G62350 & -4.26 \\
\hline XET 33 & POPTR_0014s11030 & AT1G10550 & -5.00 \\
\hline XTH 5 & POPTR_0003s15800 & AT5G13870 & -4.94 \\
\hline XTH 9 & POPTR $0019 \mathrm{~s} 14590$ & AT4G03210 & -3.61 \\
\hline XTH 28 & POPTR_0008s 13780 & AT1G14720 & -3.04 \\
\hline CesA 1 & POPTR_0018s01540 & AT4G32410 & -1.49 \\
\hline CesA 3 & POPTR_0016s05520 & AT5G05170 & -3.49 \\
\hline CesA 6 & POPTR_0007s07120 & AT5G64740 & -1.32 \\
\hline CesA 7 & POPTR_0006s 19580 & AT5G17420 & -1.75 \\
\hline CesA 8 & POPTR_0011s07040 & AT4G18780 & -2.62 \\
\hline CesA A4 & POPTR 0002s25970 & AT5G44030 & -1.39 \\
\hline CesA-like C6 & POPTR 0002s24980 & AT3G07330 & -3.68 \\
\hline CesA-like D5 & POPTR $0002 \mathrm{~s} 20130$ & AT1G02730 & -2.97 \\
\hline GSL12 & POPTR $0003 \mathrm{~s} 21690$ & AT5G13000 & -2.76 \\
\hline Laccase 2 & POPTR $0009 \mathrm{~s} 15860$ & AT2G29130 & -4.58 \\
\hline Laccase 17 & POPTR $0006 \mathrm{~s} 08740$ & AT5G60020 & -4.26 \\
\hline Peroxidase 31 & POPTR_0001s34660 & AT3G28200 & -4.37 \\
\hline PAL 2 & POPTR_0010s 23100 & AT3G53260 & 2.05 \\
\hline PAL 1 & POPTR_0016s09230 & AT2G37040 & 2.95 \\
\hline SAMS & POPTR_0013s00550 & AT3G17390 & 1.05 \\
\hline $4 C L 2$ & POPTR_0006s 18490 & AT3G21240 & 2.47 \\
\hline ССоАОМТ & POPTR_0008s 13600 & AT1G67980 & 1.67 \\
\hline $\mathrm{C} 4 \mathrm{H}$ & POPTR 0019s15110 & AT2G30490 & 1.41 \\
\hline CAD 9 & POPTR_0001s31470 & AT4G39330 & 3.13 \\
\hline$C A D$ & POPTR 0011s15120 & AT1G72680 & 1.09 \\
\hline
\end{tabular}

$\mathrm{PME}=$ pectin methylesterase; PAE $=$ pectin acetylesterase; XET: xyloglucan:xyloglucosyl transferase; $\mathrm{XTH}=$ xyloglucan endotransglucosylase/hydrolase; CesA = cellulose synthase; GSL12 = glucan synthase-like 12; PAL = phenylalanine ammonia-lyase; $\mathrm{SAMS}=\mathrm{S}$-adenosylmethionine synthetase; $4 \mathrm{CL}=4$-coumarate:CoA ligase; $\mathrm{CCoAOMT}$ = caffeoyl CoA 3-O-methyltransferase; $\mathrm{C} 4 \mathrm{H}=$ cinnamate-4-hydroxylase; $\mathrm{CAD}=$ cinnamyl alcohol dehydrogenase.

Arabinogalactan proteins (AGPs) are one of the most important cell wall structural proteins (Showalter, 2001). AGPs are highly glycosylated hydroxyproline-rich glycoproteins (HRGPs) that are expressed mostly in the plant cell wall and plasma membrane. Thus, they are believed to have functions in plant growth and development (Showalter, 2001). Our data showed that $A G P S$ were up-regulated in the newly formed xylem, suggesting their function in the earlier stage of xylem development. Fasciclin-like arabinogalactan proteins (FLAs) are a subclass of AGPs, which have glycosylated regions and cell adhesion domains (Gaspar et al., 2001). Our results showed that $F L A 1,4,6,7,10$, and 17 were up-regulated in the newly formed xylem; on the other hand, FLA12 showed preferential expression in the lignified xylem. Glycine-rich proteins (GRPs) and proline-rich proteins (PRPs) are other two important cell wall structural proteins. In this study, $P R P S$ were exclusively up-regulated in the newly formed xylem. In contrast, different GRPS were preferentially expressed in either newly formed or lignified xylem. HRGPS also showed a similar expression pattern as that of GRPS. 


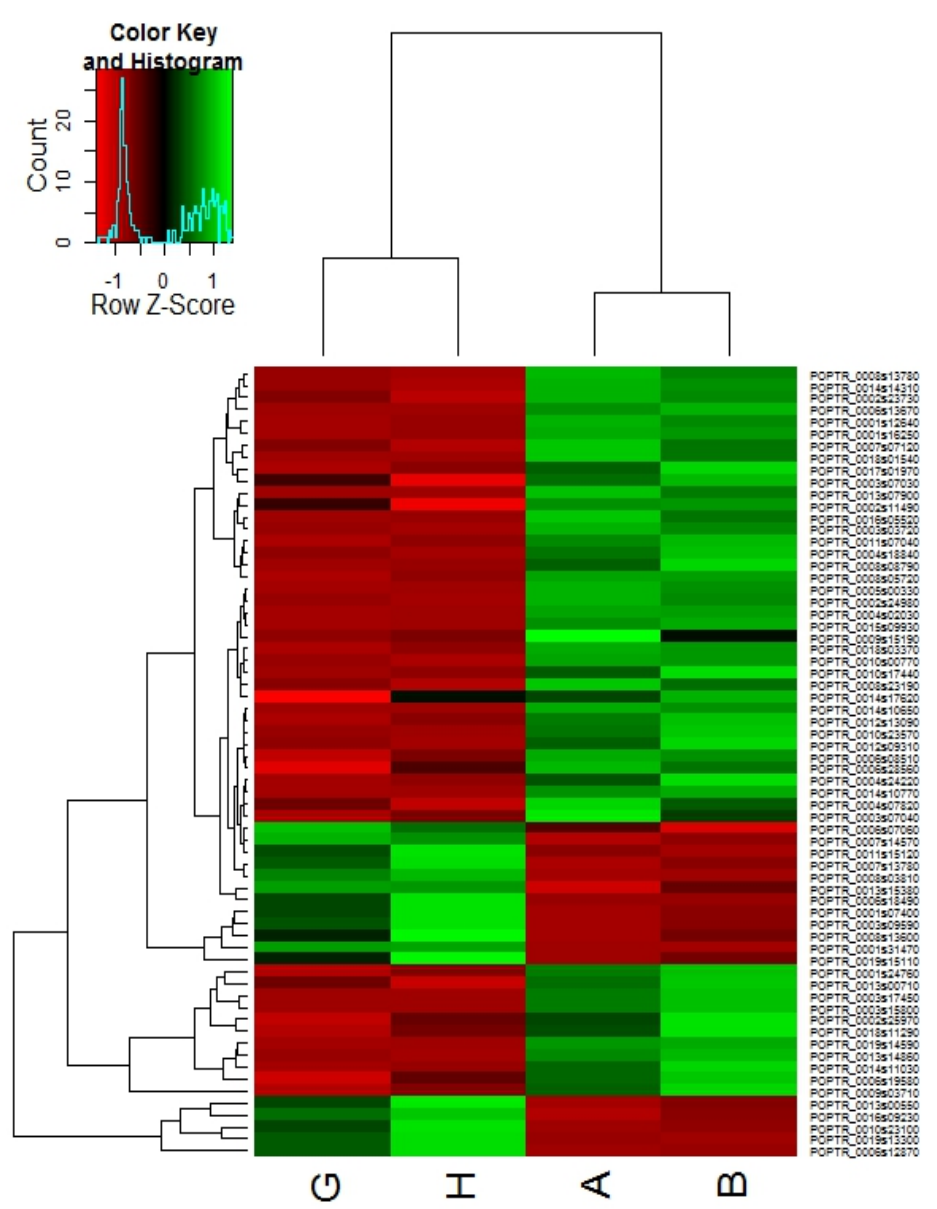

Figure 4. Heat map of differentially expressed genes involved in cell wall biosynthesis.

Many genes involved in primary wall formation were up-regulated in the newly formed xylem (Table 3). Expansin actively expressed in the cambial region had functions in the expansion of the primary cell walls of hybrid aspen (Gray-Mitsumune et al., 2004). Expansin superfamily contains expansin A, B, and expansin-like A and B. Our results showed that expansin A and B were preferentially expressed in the newly formed and lignified xylem, respectively (Table 3 ), suggesting their divergent roles in secondary xylem differentiation. Genes involved in cellulose biosynthesis (cellulose synthase, cellulose synthase-like, and glucan synthase-like 12) were up-regulated in the newly formed xylem. Some of these cellulose synthases are secondary cell wall genes; thus, the newly formed xylem underwent active cellulose synthesis during both primary and secondary wall formation. Overall, the newly formed xylem sampled in summer (July) could include various secondary xylem cells at the stages of cell division, cell expansion, primary wall synthesis, and cellulose synthesis of secondary walls. In fact, the newly formed xylem sampled in this study contained some vascular cambium cells that had undergone cell division. 
Lignin is a complex aromatic heteropolymer that is mainly deposited in the thickened secondary cell walls, and it is a major determinant of stem stiffness and pathogen resistance. Lignification is the most important symbol of secondary cell wall deposition in forest trees. Many genes involved in lignin biosynthesis were up-regulated in the lignified xylem tissues, such as $C 4 H, 4 C L, C A D, P A L, C C O A O M T 1$, and SAMS. These results suggested that the lignified xylem was at the developmental stage of lignification and deposition of secondary walls.

\section{Differential expression of transcription factors}

The transcription factor (TF) database (PlantTFDB; Zhang et al., 2011) revealed a total of $44 \mathrm{TF}$ families with differential expression in the newly formed and lignified xylem, accounting for $75.9 \%$ of the total TFs identified in the $P$. trichocarpa genome. These TF families included 112 and 312 genes differentially expressed in the newly formed and lignified xylem, respectively (Table 4). Most of the identified TF families were up-regulated in the lignified xylem, including TALE (7.1\%), bZIP (6.7\%), ERF (6.1\%), MYB_related (6.1\%), ARF (5.1\%), C3H (4.2\%), HD-ZIP (4.2\%), Trihelix (4.2\%), G2-like (3.2\%), FARI (2.9\%), and EIL (2.2\%). On the other hand, only a fewer TF families were more highly expressed in the newly formed xylem, such as $\operatorname{WOX}(3.6 \%)$.

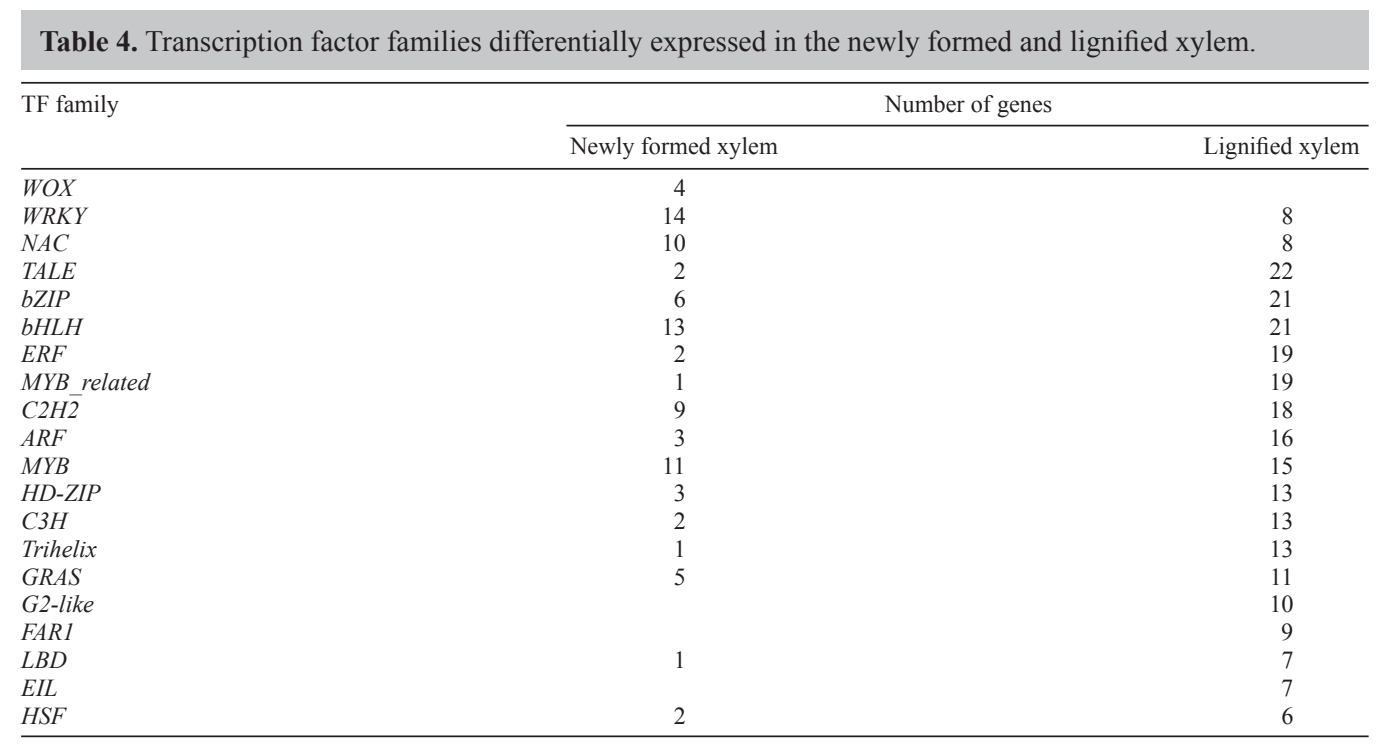

$\mathrm{TF}=$ transcription factor.

Several TF families have been shown to have roles in wood formation, such as $\mathrm{C} 2 \mathrm{H} 2$, $C 3 H, b H L H$, bZIP, NAC, MYB-related, MYB, WRKY, Thrihelix, and ELI (Scarpella and Meijer, 2004; Li et al., 2009b). Transgenic study and quantitative trait loci analysis revealed that MYBs play a role in both lignin biosynthesis and wood formation (Goicoechea et al., 2005). In the present study, MYBs were differentially expressed in the newly formed and lignified xylem, respectively, suggesting their diverse roles in the different stages of secondary xylem 
differentiation. Different members of $N A C$ were also differentially expressed in the newly formed and lignified xylem. $N A C(N A M / A T A F / C U C)$ is highly expressed in developing xylem and differentiating tracheids (Demura et al., 2002; Kubo et al., 2005). Some other NAC genes are known to be important regulators of wood formation and show higher expression in vascular tissues (Kubo et al., 2005; Mitsuda et al., 2007). Furthermore, NACs (SND1, NST1, NST2, and NST3) are involved in the regulation of fiber cell differentiation and secondary wall formation (Zhong et al., 2006; Mitsuda et al., 2007; Mitsuda and Ohme-Takagi, 2008).

\section{Differential expression of hormone-related genes}

Many auxin-related genes were up-regulated in the newly formed xylem (Table 5), such as AUX/IAA transcriptional regulator, auxin efflux carrier, auxin-like 1 protein, auxinresponsive protein, and SAUR-like auxin-responsive protein. However, genes encoding auxin response factor $(A R F 2,9,10,16,17)$ were preferentially expressed in the lignified xylem. Thus, different auxin signaling genes might have diverse functions in the regulation of secondary xylem differentiation. The diverse functions might be important for maintaining auxin balance and responding to auxin stimulus (Golisz et al., 2008; Péret et al., 2012). Genes that were more transcribed in the lignified xylem might have a function in calcium ion transport and response to auxin stimulus (Hagen and Guilfoyle, 2002), whereas auxin-related genes that were preferentially expressed in the newly formed xylem were likely to have a role in cell wall modification in response to auxin (Tamaoki et al., 2008).

\begin{tabular}{|c|c|c|c|}
\hline Gene & Poplar gene model & TAIR annotation & $\begin{array}{c}\log _{2} \text { ratios } \\
\text { (newly formed xylem/lignified xylem) }\end{array}$ \\
\hline AUX/IAA transcriptional regulator & POPTR_0013s03860 & AT5G43700 & -2.14 \\
\hline Auxin efflux carrier protein & POPTR_0011s14860 & AT1G76520 & -3.92 \\
\hline Auxin efflux carrier protein & POPTR_0016s03450 & AT1G73590 & -3.14 \\
\hline Auxin response factor 1 & POPTR_0004s23770 & AT1G59750 & -1.13 \\
\hline Auxin-like 1 protein & POPTR_0002s03520 & AT1G75310 & -1.60 \\
\hline Auxin-responsive protein & POPTR_0002s25080 & AT3G25290 & -1.26 \\
\hline Auxin-responsive protein & POPTR_0014s15980 & AT2G04850 & -2.01 \\
\hline Auxin-responsive protein & POPTR_0002s25070 & AT3G07390 & -4.58 \\
\hline Auxin-responsive GH3 protein & POPTR_0009s09590 & AT2G14960 & -3.37 \\
\hline SAUR-like auxin-responsive protein & POPTR_0005s09920 & AT3G12830 & -1.13 \\
\hline SAUR-like auxin-responsive protein & POPTR_0006s13940 & AT5G20810 & -2.12 \\
\hline SAUR-like auxin-responsive protein & POPTR_0001s16460 & AT1G72430 & -1.99 \\
\hline SAUR-like auxin-responsive protein & POPTR_0009s12880 & AT1G75590 & -1.51 \\
\hline Indole-3-acetic acid inducible 14 & POPTR_0010s08880 & AT4G14550 & -3.24 \\
\hline Indole-3-acetic acid inducible 29 & POPTR_0006s27130 & AT4G32280 & -2.94 \\
\hline Indole acetic acid-induced protein 16 & POPTR_0005s05560 & AT3G04730 & 1.16 \\
\hline Auxin response factor 2 & POPTR_0015s11660 & AT5G62000 & 1.43 \\
\hline Auxin response factor 9 & POPTR_0003s14200 & AT4G23980 & 1.59 \\
\hline Auxin response factor 10 & POPTR_0009s02020 & AT2G28350 & 2.19 \\
\hline Auxin response factor 11 & POPTR_0002s17350 & AT2G46530 & 2.93 \\
\hline Auxin response factor 16 & POPTR_0006s12930 & AT4G30080 & 2.95 \\
\hline Auxin response factor 17 & POPTR_0002s09050 & AT1G77850 & 2.36 \\
\hline Auxin-responsive protein & POPTR_0014s16010 & AT5G47530 & 1.75 \\
\hline Auxin-responsive GH3 protein & POPTR_0014s09120 & AT2G46370 & 2.06 \\
\hline SAUR-like auxin-responsive protein & POPTR_0002s17700 & AT2G46690 & 2.37 \\
\hline Ethylene-forming enzyme & POPTR_0014s15710 & AT1G05010 & -3.01 \\
\hline Ethylene insensitive 3 protein & POPTR_0008s01200 & AT3G20770 & 1.47 \\
\hline Ethylene response sensor 1 & POPTR_0002s20260 & AT2G40940 & 1.60 \\
\hline ERE binding factor 1 & POPTR_0003s 15030 & AT4G17500 & 3.82 \\
\hline ERE binding factor 4 & POPTR_0001s40770 & AT3G15210 & 1.06 \\
\hline Ethylene insensitive-like 3 & POPTR_0003s22090 & AT1G73730 & 1.46 \\
\hline
\end{tabular}


A gene encoding ethylene-forming enzyme was up-regulated in the newly formed xylem, whereas genes encoding ethylene signaling enzymes were more transcribed in the lignified xylem (Table 5). These results indicated that ethylene signals could be involved in secondary xylem differentiation. Ethylene insensitive 3 and ethylene insensitive-like 3 are also transcription factors that play an essential role in higher plant growth and development and stress resistant, as well as programmed cell death (Chao et al., 1997).

\section{Differential expression of cell cycle-related genes}

Cell proliferation is regulated by several proteins such as cyclins, cyclin-dependent kinase (CDKs), and cyclin-dependent kinase inhibitors (CKIs; Hong et al., 1998; Hattori et al., 2000; Meijer and Murray, 2001). In the present study, CDKs and cyclins were up-regulated in the newly formed xylem, whereas $C K I$, an inhibitor of cyclin, was preferentially expressed in the lignified xylem (Table 6). The results suggested that cell division process is more active in the newly formed xylem, and it was inhibited in the lignified xylem by the expression of CKI genes.

\begin{tabular}{|c|c|c|c|c|}
\hline \multirow[t]{2}{*}{ Gene } & \multirow[t]{2}{*}{ Poplar gene model } & \multirow[t]{2}{*}{ TAIR annotation } & \multicolumn{2}{|c|}{$\log _{2}$ ratios } \\
\hline & & & Newly formed xylem & Lignified xylem \\
\hline cyclin $A 1 ; 1$ & POPTR_0005s20380 & AT1G44110 & -2.69 & \\
\hline cyclin D $3 ; 1$ & POPTR_0014s02310 & AT4G34160 & -3.61 & \\
\hline cyclin D $3 ; 2$ & POPTR_0005s 18550 & AT5G67260 & -3.37 & \\
\hline cyclin & POPTR_0005s03620 & AT3G05330 & -1.90 & \\
\hline cyclin $p 3 ; 2$ & POPTR_0002s14440 & AT3G60550 & -1.12 & \\
\hline cyclin $p 4 ; 1$ & POPTR_0014s04930 & AT2G44740 & -2.77 & \\
\hline$C D K B 1 ; 2$ & POPTR_0006s11390 & AT2G38620 & -2.64 & \\
\hline$C D K B 2 ; 1$ & POPTR_0005s27890 & AT1G76540 & -2.92 & \\
\hline Inhibitor with $C D K$ & POPTR_0001s32120 & AT5G48820 & & 1.61 \\
\hline Cycling DOF factor 2 & POPTR_-0017s12080 & AT5G39660 & & 1.48 \\
\hline
\end{tabular}

$C D K=$ cyclin-dependent kinase.

\section{Validation of microarray gene expression by using real-time RT-PCR}

Eight differentially expressed genes were selected for the validation by using real-time RT-PCR. The RT-PCR results are reported as $\log _{2}$ ratios of gene expression in the newly formed xylem compared to those in the lignified xylem. Differential gene transcription (y-axis, $\log _{2}$ ratio) was shown for each selected gene (x-axis; Figure 5. $\mathrm{x}$-axis: eight genes involved in the validation, including phosphatase 2C (POPTR_0013s01370), patatin-like protein 9 (POPTR_0005s23170), basic chitinase (CV277108), unknown protein (POPTR_0008s14970), thaumatin (POPTR_0009s13510), expansin-A4 (POPTR_0008s05720), expansin-A1 (POPTR_0008s08790), and expansinA8 (POPTR_0013s15080); y-axis: $\log _{2}$ ratio of gene expression in the newly formed xylem compared to the lignified xylem.). No significant differences were observed between the RT-PCR and microarray gene expression results. Therefore, differentially expressed genes identified by microarray experiments in this study were relatively reliable for the transcriptome comparison during secondary xylem differentiation. 


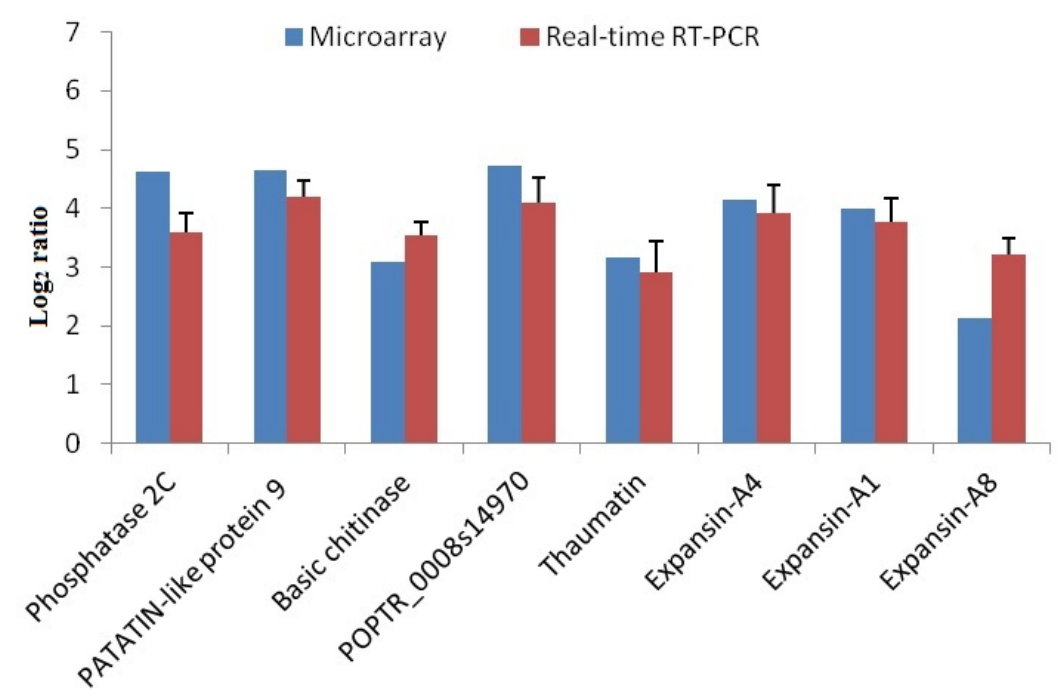

Figure 5. Validation of microarray gene expression by real-time reverse transcriptase-polymerase chain reaction.

\section{CONCLUSION}

The Affymetrix Poplar Genome Chips identified 6843 genes (approximately $11 \%$ of the total probes) with differential expression in the newly formed and lignified xylem in $P$. tomentosa. Many genes that were up-regulated in the newly formed xylem had roles in cell division, primary wall formation, and cellulose synthesis of secondary walls, such as cyclins, CDKs, expansin, XHT, XETs, pectin acetylesterase, pectin methylesterase, and several cellulose synthases. In contrast, many genes, including $C 4 H, 4 C L, C A D, P A L, C C O A O M T 1$, and $S A M S$, involved in lignin biosynthesis were highly transcribed in the lignified xylem. Many transcription factors were up-regulated in the lignified xylem, suggesting that extensive transcription regulation occurs in this tissue. Furthermore, hormone-related genes were differentially expressed in the two developing xylem tissues; thus, hormone signaling genes play important roles during secondary xylem differentiation.

\section{ACKNOWLEDGMENTS}

Research supported by grants from the Forestry Public Benefit Research Program (\#201304102), the State Key Basic Research Program of China (\#2012CB114506), and the Project of the National Natural Science Foundation of China (\#31170622, \#30872042). Yuanzhen Lin was thanked for the help on data analysis.

\section{Supplementary material}

\section{REFERENCES}

Bao YH, Dharmawardhana P, Mockler TC and Strauss SH (2009). Genome scale transcriptome analysis of shoot organogenesis in Populus. BMC Plant Biol. 9: 132. 
Chao Q, Rothenberg M, Solano R, Roman G, et al. (1997). Activation of the ethylene gas response pathway in Arabidopsis by the nuclear protein ETHYLENE-INSENSITIVE3 and related proteins. Cell 89: 1133-1144.

Cutter EG (1965). Recent experimental studies of the shoot apical bud and shoot morphogenesis. Bot. Rev. 31: 7-113.

Demura T, Tashiro G, Horiguchi G, Kishimoto N, et al. (2002). Visualization by comprehensive microarray analysis of gene expression programs during transdifferentiation of mesophyll cells into xylem cells. Proc. Natl. Acad. Sci. U. S. A. 99: 15794-15799.

Du Z, Zhou X, Ling Y, Zhang ZH, et al. (2010). AgriGO: a GO analysis toolkit for the agricultural community nucleic acids research advance access. Nucleic Acids Res. 38: W64-W70.

Gaspar Y, Johnson KL, McKenna JA, Bacic A, et al. (2001). The complex structures of arabinogalactan-proteins and the journey towards understanding function. Plant Mol. Biol. 47: 161-176.

Girke T, Lauricha J, Tran H, Keegstra K, et al. (2004). The Cell Wall Navigator database. A systems-based approach to organism-unrestricted mining of protein families involved in cell wall metabolism. Plant Physiol. 136: 3003-3008.

Goicoechea M, Lacombe E, Legay S, Mihaljevic S, et al. (2005). EgMYB2, a new transcriptional activator from Eucalyptus xylem, regulates secondary cell wall formation and lignin biosynthesis. Plant J. 43: 553-567.

Golisz A, Sugano M and Fujii Y (2008). Microarray expression profiling of Arabidopsis thaliana L. in response to allelochemicals identified in buckwheat. J. Exp. Bot. 59: 3099-3109.

Gray-Mitsumune M, Mellerowicz EJ, Abe H, Schrader J, et al. (2004). Expansins abundant in secondary xylem belong to subgroup A of the alpha-expansin gene family. Plant Physiol. 135: 1552-1564.

Hagen G and Guilfoyle T (2002). Auxin-responsive gene expression: genes, promoters and regulatory factors. Plant Mol. Biol. 49: 373-385.

Hattori N, Davies TC, Anson-Cartwright L and Cross JC (2000). Periodic expression of the cyclin-dependent kinase inhibitor p57(Kip2) in trophoblast giant cells defines a G2-like gap phase of the endocycle. Mol. Biol. Cell 11: 1037-1045.

Hertzberg M, Aspeborg H, Schrader J, Andersson A, et al. (2001). A transcriptional roadmap to wood formation. Proc. Natl. Acad. Sci. U. S. A. 98: 14732-14737.

Hong Y, Roy R and Ambros V (1998). Developmental regulation of a cyclin-dependent kinase inhibitor controls postembryonic cell cycle progression in Caenorhabditis elegans. Development 125: 3585-3597.

Kubo M, Udagawa M, Nishikubo N, Horiguchi G, et al. (2005). Transcription switches for protoxylem and metaxylem vessel formation. Genes Dev. 19: 1855-1860.

Larson PR (1994). The Vascular Cambium: Development and Structure. Springer Series in Wood Science, Springer, Berlin.

Li D, Su Z, Dong J and Wang T (2009a). An expression database for roots of the model legume Medicago truncatula under salt stress. BMC Genomics 10: 517.

Li X, Wu HX, Dillon SK and Southerton SG (2009b). Generation and analysis of expressed sequence tags from six developing xylem libraries in Pinus radiata D. Don. BMC Genomics 10: 41.

Meijer M and Murray JA (2001). Cell cycle controls and the development of plant form. Curr. Opin. Plant Biol. 4: 44-49.

Mitsuda N and Ohme-Takagi M (2008). NAC transcription factors NST1 and NST3 regulate pod shattering in a partially redundant manner by promoting secondary wall formation after the establishment of tissue identity. Plant J. 56: 768-778.

Mitsuda N, Iwase A, Yamamoto H, Yoshida M, et al. (2007). NAC transcription factors, NST1 and NST3, are key regulators of the formation of secondary walls in woody tissues of Arabidopsis. Plant Cell 19: 270-280.

Nystedt B, Street NR, Wetterbom A, Zuccolo A, et al. (2013). The Norway spruce genome sequence and conifer genome evolution. Nature 497: 584.

Oakley RV, Wang YS, Ramakrishna W, Harding SA, et al. (2007). Differential expansion and expression of alpha- and beta-tubulin gene families in Populus. Plant Physiol. 145: 961-973.

Ouyang S, Zhu W, Hamilton J, Lin H, et al. (2007). The TIGR rice genome annotation resource: improvements and new features. Nucleic Acids Res. 35: D883-D887.

Péret B, Swarup K, Ferguson A, Seth M, et al. (2012). AUX/LAX genes encode a family of auxin influx transporters that perform distinct functions during Arabidopsis development. Plant Cell 24: 2874-2885.

R Core Team (2012). R: A Language and Environment for Statistical Computing. R Foundation for Statistical Computing, Vienna. ISBN 3-900051-07-0. Available at [http://www.R-project.org/].

Ramsköld D, Kavak E and Sandberg R (2012). How to analyze gene expression using RNA-sequencing data. Methods Mol. Biol. 802: 259-274

Scarpella E and Meijer AH (2004). Pattern formation in the vascular system of monocot and dicot plant species. New Phytologist. 164: 209-242.

Showalter AM (2001). Arabinogalactan-proteins: structure, expression and function. Cell. Mol. Life Sci. 58: 1399-1417.

Sterky F, Regan S, Karlsson J, Hertzberg M, et al. (1998). Gene discovery in the wood-forming tissues of poplar: analysis 
of 5,692 expressed sequence tags. Proc. Natl. Acad. Sci. U. S. A. 95: 13330-13335.

Tamaoki M, Freeman JL and Pilon-Smits EA (2008). Cooperative ethylene and jasmonic acid signaling regulates selenite resistance in Arabidopsis. Plant Physiol. 146: 1219-1230.

Tuskan GA, Difazio S, Jansson S, Bohlmann J, et al. (2006). The genome of black cottonwood, Populus trichocarpa (Torr. \& Gray). Science 313: 1596-1604.

Wang M, Qi X, Zhao S, Zhang S, et al. (2009). Dynamic changes in transcripts during regeneration of the secondary vascular system in Populus tomentosa Carr. revealed by cDNA microarrays. BMC Genomics 10: 215.

Zhang H, Jin J, Tang L, Zhao Y, et al. (2011). PlantTFDB 2.0: update and improvement of the comprehensive plant transcription factor database. Nucleic Acids Res. 39: D1114-D1117.

Zhong R, Demura T and Ye ZH (2006). SND1, a NAC domain transcription factor, is a key regulator of secondary wall synthesis in fibers of Arabidopsis. Plant Cell 18: 3158-3170. 